\title{
TISSUE SPECIFIC EXPRESSION OF CHALCONE SYNTHASE (CHS) TRANSCRIPTS AND OVEREXPRESSION OF BrCHS IN CELL SUSPENSION CULTURES OF BOESENBERGIA ROTUNDA
}

\author{
Nurnadiah Roslan ${ }^{1 a}$, Teh Ser Huy ${ }^{2 a}$, Wong Sher Ming ${ }^{3 a}$, Norzulaani Khalid ${ }^{4 a}$ and \\ Zulqarnain Mohamed ${ }^{5 \mathrm{a}^{*}}$
}

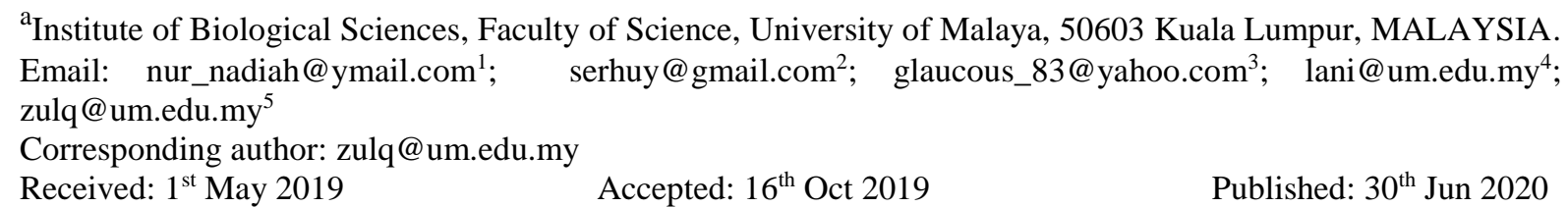
DOI: https://doi.org/10.22452/mjs.vol39no2.6

\begin{abstract}
Fingerroot (Boesenbergia rotunda) is a monocot plant from Zingiberaceae family, and it has been used as food, spices and herbal remedy for over 2000 years. Further exploration on its medicinal and health values is still on-going due to its ethnomedicinal properties derived from rhizome extracts. Over the years, compounds extracted from its rhizome have been well studied, and the findings showed that it posed anti-cancer, anti-microbial, anti-viral and anti-inflammatory properties. These compounds are plant secondary metabolites synthesized from flavonoid biosynthesis pathway by a number of enzymes. In most plants, chalcone synthase (CHS) is one of the key enzymes that initiate the flavonoid biosynthesis pathway. The aim of this research is to overcome the low yield of most of the important compounds that are extracted from rhizomes through exploiting the richness of biochemistry by metabolic engineering and applying suspension culture as a plant host. In this study, at least four to five copies of $\mathrm{BrCHS}$ transcripts were discovered and found to be predominantly expressed in a tissue-specific manner (leaves, flowers, rhizomes and cell suspension cultures). Later, $\mathrm{BrCHS} 2$ transcript isolated from the rhizomes was selected to be introduced into $B$. rotunda cell suspension culture to observe the regulation of transgene expression in the plant pathways. The findings of this study will rebound to the benefit of researchers in utilizing important plant genes that could give a clue to exploit the synthesis of targeted compounds, and to explore the genetic control of plants in a particular pathway.
\end{abstract}

Keywords: Boesenbergia rotunda, Chalcone synthase, Overexpression, Pinocembrin chalcone

\section{INTRODUCTION}

Boesenbergia rotunda is a herb plant from the Boesenbergia genera, and belongs to the Zingiberaceae family. It is widely cultivated throughout Southeast Asia, where it grows in dense forest and shaded lowland areas. It is a type of ginger species which is botanically known as
Boesenbergia rotunda (L.) Mansf., and commonly recognized as Chinese keys or Fingerroot in English, "Temu Kunci" in Malay and "Krachai" or "Krachai-Dang" in Thailand. Most people in many Asian countries use this fingerroot plant as condiment in food due to its aromatic flavour. Traditionally, fresh rhizomes are practically used in tonic mixture for women 
after childbirth as a stomachic to improve digestion and as a remedy for coughs, wounds and mouth ulcers (Tewtrakul et al., 2009). The popularity of its ethnomedicinal usage that is particularly derived from rhizome extracts has drawn wide attention for researchers to further explore its pharmaceutical values that could therefore explain the significance of its traditional usage.

Over the years, researchers have successfully isolated and elucidated nearly a hundred of bioactive compounds extracted from different parts of $B$. rotunda such as leaves, roots, stems and rhizomes. An array of bioactive compounds of $B$. rotunda was shown to execute medicinal benefits as it has exhibited antiviral (Cheenpracha et al., 2006; Kiat et al., 2006), antimicrobial (Bhamarapravati et al., 2006), antioxidant (Shindo et al., 2006) and anticancer (Kirana et al., 2007) activities. With the various pharmaceutical potential and values of bioactive compounds found in B. rotunda plants, chalcone synthase (CHS) has become the most exclusively studied enzymes as it generates the backbones of a variety of plant secondary metabolites in flavonoid biosynthesis pathways. CHS performs as a starting enzyme that catalyses sequential condensation and decarboxylation reactions of two substrates (malonyl-CoA and $p$-Coumaroyl-CoA), and creates fifteen structures of backbone from which all different flavonoids are derived from (Falcone Ferreyra et al., 2012; Shashank \& Abhay, 2013; Sun et al., 2015; Deng et al., 2018).

Chalcone synthase (CHS; EC 2.3.1.74) belongs to the CHS superfamily of plant type III polyketide synthases (PKSs) which resembles closely the condensing enzyme of type-II fatty acid synthesis with respect to several of its catalytic and structural properties (Austin $\&$ Noel, 2003). Since the first isolation of CHS gene from parsley (Petroselium hortense) by Kreuzaler in 1983, the study of this enzyme is now rapidly progressing with more than 2000 CHS protein from different plant species being successfully cloned and sequenced according to NCBI RefSeq database (O'Leary et al,. 2016). Based on previous literature, only a single copy of the CHS gene is found in Arabidopsis thaliana (Feinbaum \& Ausubel, 1992). Plant species such as Gerbera hybrida (Deng et al., 2014) and Ipomea purpurea (Durbin et al., 2000) contain small number of $C H S$ gene families with 3 and 6, respectively. Meanwhile, a total of 14 members of $C H S$ gene family was identified in Zea mays L. (Han et al., 2016), and recently, 14 unique GmCHS (GmCHS1-GmCHS14) were found in Glycine $\max$ (Anguraj Vadivel et al., 2018).

Previously, in-vitro propagation method with morphogenetic potential and growth capacity from cell suspension cultures of B. rotunda were explored as an alternative source of flavonoid compounds, namely alpinetin, pinocembrin, cardamonin, pinostrobin and panduratin A (Wong et al., 2013; Yusuf et al., 2013). Therefore, the propagation of $B$. rotunda cell suspension cultures can be scaled up to yield valuable compounds due to its homogeneity, and has high rate of cell growth under controlled condition (Rasche et al., 2016). However, due to the limitation report on its number of $C H S$ genes, it is vital to characterize and analyse those genes from different plant sources. With several advantages of utilizing cell suspension cultures as plant host, this study further provides the idea of enhancing the accumulation of targeted compounds 
through metabolic engineering by overexpressing the targeted $\mathrm{BrCHS}$.

\section{MATERIALS AND METHODS}

\subsection{Plant Materials}

The $B$. rotunda samples were collected from a field located at Temerloh, Pahang (GPS:3.636385, 102.415142). The leaves, flowers and rhizomes were frozen immediately in liquid nitrogen, and stored at $-80^{\circ} \mathrm{C}$ until further usage. Fine cell suspension culture was established from a single bud that emerged from the rhizome, and propagated in Murashige and Skoog (MS) (Murashige \& Skoog, 1962) with 1mg/L 2,4-dichlorophenoxyacetic acid (2,4-D) and 0.5mg/L 6-benzylaminopurine (BAP) (Wong et al., 2013). Subculture was carried out at every 10 days.

\section{$2.2 \quad$ Assessment of BrCHS}

Genomic DNA was isolated from the leaves of $B$. rotunda according to the method described by Khayat et al. (2004). Partial BrCHS was amplified using degenerated primers designed to target the gene's core fragment. Later, PCR products were purified (QIAquick Gel Extraction kit, UK) and cloned into E. coli strain JM109 via pGEM-T Easy vector (Promega, USA). DNA sequencing was then performed for each positive transformant colony. Conserved regions of the $\mathrm{BrCHS}$ was used to design gene specific primers for $5^{\prime}$ and 3' Rapid Amplification cDNA ends (RACE). Total RNA was isolated from the leaves, flowers, rhizomes and cell suspension cultures of $B$. rotunda (Chan et al., 2007). RNA samples were treated with DNaseI (Invitrogen, USA), and used as template in 5' and 3' RACE amplification using GeneRacer kit (Invitrogen, USA) and various combinations of gene specific primers (Suppl. 1). The PCR products were again cloned into pGEM-T Easy vector System (Promega, USA). DNA sequencing was carried out using BigDye ${ }^{\circledR}$ Terminator v3.1 Cycle Sequencing Kit on the $3130 x \mathrm{x}$ Genetic Analyzer (Applied Biosystem, USA). The sequences were analysed and aligned using Mega 6 software. For Southern blot analysis, a total of $10 \mu \mathrm{g}$ genomic DNA was digested with EcoRI (Promega, USA), HindIII (NEB, UK), DraI (Promega, USA) and EcoRV (Promega, USA), respectively. The digested DNA was fractionated on $0.7 \%$ agarose gel, and blotted onto Hybond $\mathrm{N}^{+}$nylon membrane (GE Healthcare, USA). The CHS coding region was used as the probe. The labelling of the probe, prehybridization, hybridization and detection were done according to the protocol of DIG High Prime DNA Labelling and Detection Starter Kit (Roche, USA) (Sambrook et al., 1989).

\subsection{Cloning of BrCHS2 into E. coli (pCAMBIA1304 vector system)}

For the purpose of transforming the $\mathrm{BrCHS}$ in cell suspension cultures, $\mathrm{BrCHS}$ that was predominantly expressed in rhizome was chosen and referred to as BrCHS2 transcript. The pGEM-T easy vector harbouring the entire coding region of $\mathrm{BrCHS} 2$ transcript was digested with restriction endonucleases, NcoI (Fermentas, USA) and SpeI (Fermentas, USA), followed by purification, and ligation into pCAMBIA-1304 (GenBank accession no. AF234300.1) vector system.

\subsection{Plant infection, co-cultivation and selection}

B. rotunda cell suspension cultures were used as the target explant in this 
project. Transformed Agrobacterium carrying recombinant construct was cultured overnight for 16 to 18 hours in LB broth medium containing $30 \mathrm{mg} / \mathrm{mL}$ of Rifampicin and $50 \mathrm{mg} / \mathrm{L}$ of Kanamycin. Approximately, $2 \mathrm{~mL}$ of settled cell volume (SCV) of cell suspension cultures were briefly air-dried by sucking out the liquid media and left in laminar flow cabinet for 10 to $15 \mathrm{~min}$. Cells were infected by bacteria suspensions at room temperature for $10 \mathrm{~min}$ without shaking. During the infection, $100 \mu \mathrm{M}$ of Acetosyringone (AS) was added to the MS liquid medium. After the infection, the cells were blotted dry on filter paper in a petri dish containing solid MS medium supplemented with $1 \mathrm{mg} / \mathrm{L}$ of $2,4-\mathrm{D}, 0.5 \mathrm{mg} / \mathrm{L}$ of BAP and $100 \mu \mathrm{M}$ of AS. The cells were then co-cultivated for 3 days in dark condition. Next, the infected cells were cultured in liquid MS medium supplemented with $1 \mathrm{mg} / \mathrm{L}$ of $2,4-\mathrm{D}$, $0.5 \mathrm{mg} / \mathrm{L}$ of BAP and $300 \mathrm{mg} / \mathrm{L}$ of Cefotaxime (Duchefa Biochemie, Netherlands) on a rotary shaker (100rpm) at $25 \pm 1^{\circ} \mathrm{C}$ for 1 week. The cells were then grown on semi-solid MS medium supplemented with $1.0 \mathrm{mg} / \mathrm{L}$ of $2,4-\mathrm{D}$, $0.5 \mathrm{mg} / \mathrm{L}$ of BAP, $300 \mathrm{mg} / \mathrm{L}$ of Cefotaxime and $15 \mathrm{mg} / \mathrm{L}$ of Hygromycin (Duchefa Biochemie, Netherlands) for selection of resistant callus in 4-week intervals, respectively.

\subsection{Screening of Transgenic Cell Suspension Cultures}

After a few rounds of screening selection with hygromycin and $\beta$ glucuronidase (GUS) assay, a few clumps of resistant callus that showed positive screening were selected and transferred to liquid MS media. The subculture of cell suspension cultures was then carried out every ten days up to two months to acquire sufficient amount of cell cultures. Next, after two months of culture, the cells were then harvested and tested with $\beta$ glucuronidase (GUS). DNA extraction on cells with positive GUS assay was carried out as described by Khayat et al. (2004) to perform multiplex PCR using two sets of primers: (i) CHS_F and pCAMBIA_R; to detect the recombinant $\mathrm{BrCHS} 2$ and (ii) HptII_F and HptII_R; to detect hygromycin resistant gene. Elongation factor $1 \alpha(E F 1 \alpha)$ gene was used as the internal control. The primers' sequences were listed in Suppl. 1.

\subsection{Reverse Transcription Quantitative Real-Time PCR (RT-qPCR)}

A total of $250 \mathrm{mg}$ of cells from suspension cultures (either transgenic or wildtype) was used for the RNA extraction as described by Chan et al. (2007). The RNA samples were treated with DNaseI (Invitrogen, USA) at $37^{\circ} \mathrm{C}$ for $15 \mathrm{~min}$ to remove residual genomic DNA, and RNA concentrations were determined using a NanoPhotometer (IMPLEN, Germany). The one step RT-qPCR was carried out using Power SYBR ${ }^{\circledR}$ Green RNA-to- $\mathrm{C}_{\mathrm{T}}{ }^{\mathrm{TM}}$ 1-Step kit (Applied Biosystem, USA), 100ng of total RNA as template and 100nM of each primer (CHS_rt_F and CHS_rt_R) according to manufacturer's protocol. RTqPCR was performed on a QuantStudio ${ }^{\mathrm{TM}}$ 12K Flex system (Applied Biosystem, USA) and the program was set as follows: a cycle of $48^{\circ} \mathrm{C}$ for $30 \mathrm{~min}$, a cycle of $95^{\circ} \mathrm{C}$ for $10 \mathrm{~min}$ and 40 cycles of $95^{\circ} \mathrm{C}$ for $15 \mathrm{~s}$ and $60^{\circ} \mathrm{C}$ for $1 \mathrm{~min}$. Elongation factor $1 \alpha$ (EFla) gene was used as the endogenous control and three technical replicates were included in this experiment. The fold change of expression was calculated using the comparative $C_{T}$ method $\left(2^{-\Delta \Delta C t}\right)$, where $\Delta \Delta \mathrm{Ct}=\Delta \mathrm{Ct}$ (transgenic sample endogenous control) / $\Delta \mathrm{Ct}$ (reference sample - endogenous control) where reference sample was wildtype cell suspension culture. 


\subsection{Statistical Analysis}

The experimental data for RTqPCR was subject to the one-way ANOVA analysis using the IBM SPSS Statistics 21.0 software. The tests of significance were done using Tukey's Multiple Range Test.

\section{RESULTS}

Using different combinations of primers (Figure 1 and Suppl. 1), full-length of $\mathrm{BrCHSs}$ was obtained from four different sources (leaf, flower, rhizome and cell suspension) of $B$. rotunda. More than 10 clones were sequenced from each source, giving a total of $62 \mathrm{BrCHSs}$, and were analysed. It was noted that the variabilities between the clones were the same. These variabilities can be preliminarily grouped

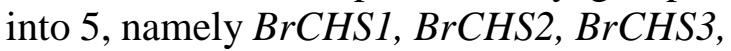
BrCHS4 and BrCHS5 (data not shown). The lengths of the coding sequence (CDS) were consistent (1176 bp), while the lengths of the untranslated regions (UTR) varied ranging between 55 and $64 \mathrm{bp}$ for 5'UTR and 52 to $178 \mathrm{bp}$ for 3'UTR (data not shown). The $B r C H S$ isolated from $B$. rotunda plants contained one intron and two exons with the presence of single intron inserted in the first Cysteine (Cys) codon, and was consistent with the result of previous studies such as in turmeric (Wannapinpong et al., 2013), crabapple (Tai et al., 2014) and citrus (Wang et al., 2018).

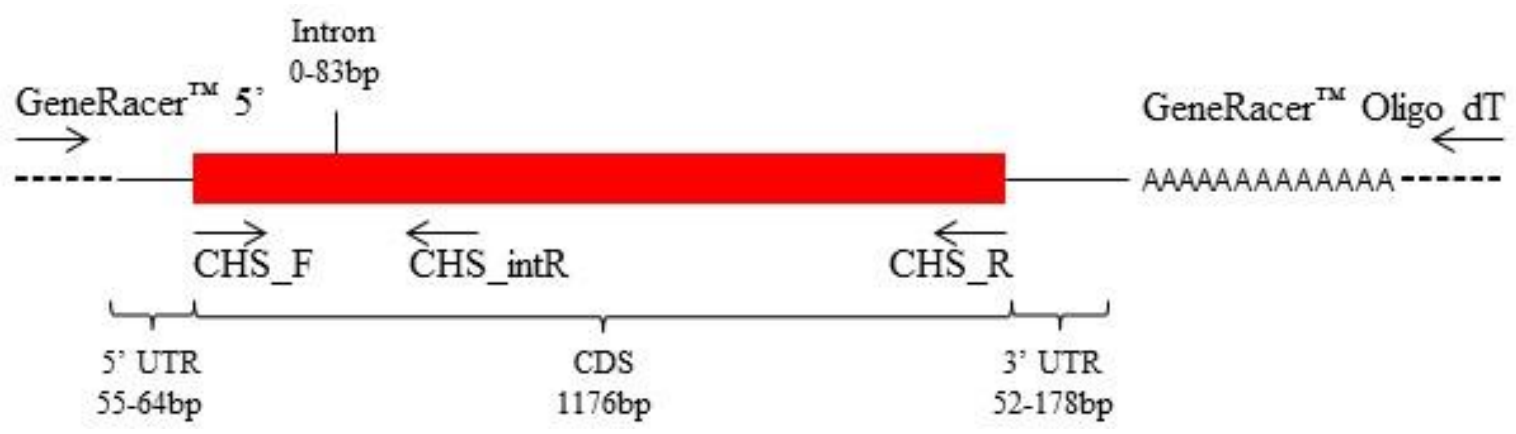

Figure 1.The schematic diagram of $\mathrm{BrCHS}$ cDNA and the binding sites of primers

The most common sequence from each group was then aligned with each other to assess genetic related study. Amino acid alignments were built and compared between five sequences and also with other plants CHS (Suppl. 2). Based on the amino acid alignment, there were several conserved regions identified: (a) Cys164His303-Asn336 as a signature sequence for CHS-like enzymes (Ferrer et al., 1999;
Resmi \& Soniya, 2012), (b) a highly conserved Pro in the GFGP375G loop which was a unique residue for CHS superfamily enzymes (Ayabe et al., 2010; Suh et al., 2000) and (c) active site residues for CHS: Phe215 and Phe265 (Wani et al., 2017; Sun et al., 2015; Abe et al., 2003; Jez et al., 2002). Together, these residues represented the domains for active sites and were important to serve as a scaffold during cyclization reaction. 
Sequence variation between five BrCHSs showed a high percentage of similarity ranging from $97.0 \%$ to $99.1 \%$ between nucleotides, and $94.3 \%$ to $99.0 \%$ between amino acids as shown in Table 1. Both nucleotide and amino acid alignments showed that $\mathrm{BrCHS} 1$ and $\mathrm{BrCHS} 2$ shared the highest similarity with only 10 mismatched nucleotides, which were causing differences in 4 amino acids in the coding of DNA sequence (CDS). Meanwhile, BrCHS3 and BrCHS4 that were differently grouped under the same plant sources, suspension cultures, showed that there were differences in 22 amino acids in the CDS.

Table 1. The percentage similarity of nucleotides and amino acids among $\mathrm{BrCHSs}$

BrCHS1 (\%) $\quad$ BrCHS2 (\%) BrCHS3 (\%) $\quad$ BrCHS4 (\%)

Nuc. A.a. Nuc. A.a. Nuc. A.a. Nuc. A.a.

\begin{tabular}{lcccccccc}
\hline $\operatorname{BrCHS} 1(\%)$ & - & - & - & - & - & - & - & - \\
\hline $\operatorname{BrCHS} 2(\%)$ & 99.1 & 99.0 & - & - & - & & - & - \\
\hline $\operatorname{BrCHS3}(\%)$ & 97.0 & 94.3 & 97.4 & 95.4 & - & & - & - \\
\hline $\operatorname{BrCHS} 4(\%)$ & 98.0 & 96.9 & 98.3 & 98.0 & 96.6 & 94.4 & - & - \\
\hline BrCHS5 (\%) & 97.6 & 95.6 & 98.0 & 96.7 & 97.0 & 95.4 & 97.8 & 96.2 \\
\hline
\end{tabular}

Nuc.: Nucleotides, A.a.: Amino acids

The amino acid alignments of 62 $\mathrm{BrCHSs}$ clones were subject to phylogenetic analysis to verify the BrCHS groups. According to phylogenetic analysis, all $\mathrm{BrCHSs}$ were branched differently as shown in Figure 2. These $\mathrm{BrCHSs}$ were predominantly expressed in different sources of the plants; therefore, this cluster result corroborated with the earlier result on the characterization of 5
BrCHS groups, and they were further characterized as five different $\mathrm{BrCHS}$ transcripts. Based on the cluster results, one $\mathrm{BrCHS}$ transcript was predominantly transcribed in leaf $(B r C H S 1)$, rhizome (BrCHS2) and flower (BrCHS5), while two $\mathrm{BrCHS}$ transcripts were predominantly transcribed in suspension cultures $(\mathrm{BrCHS} 3$ and $\mathrm{BrCHS4}$ ). 


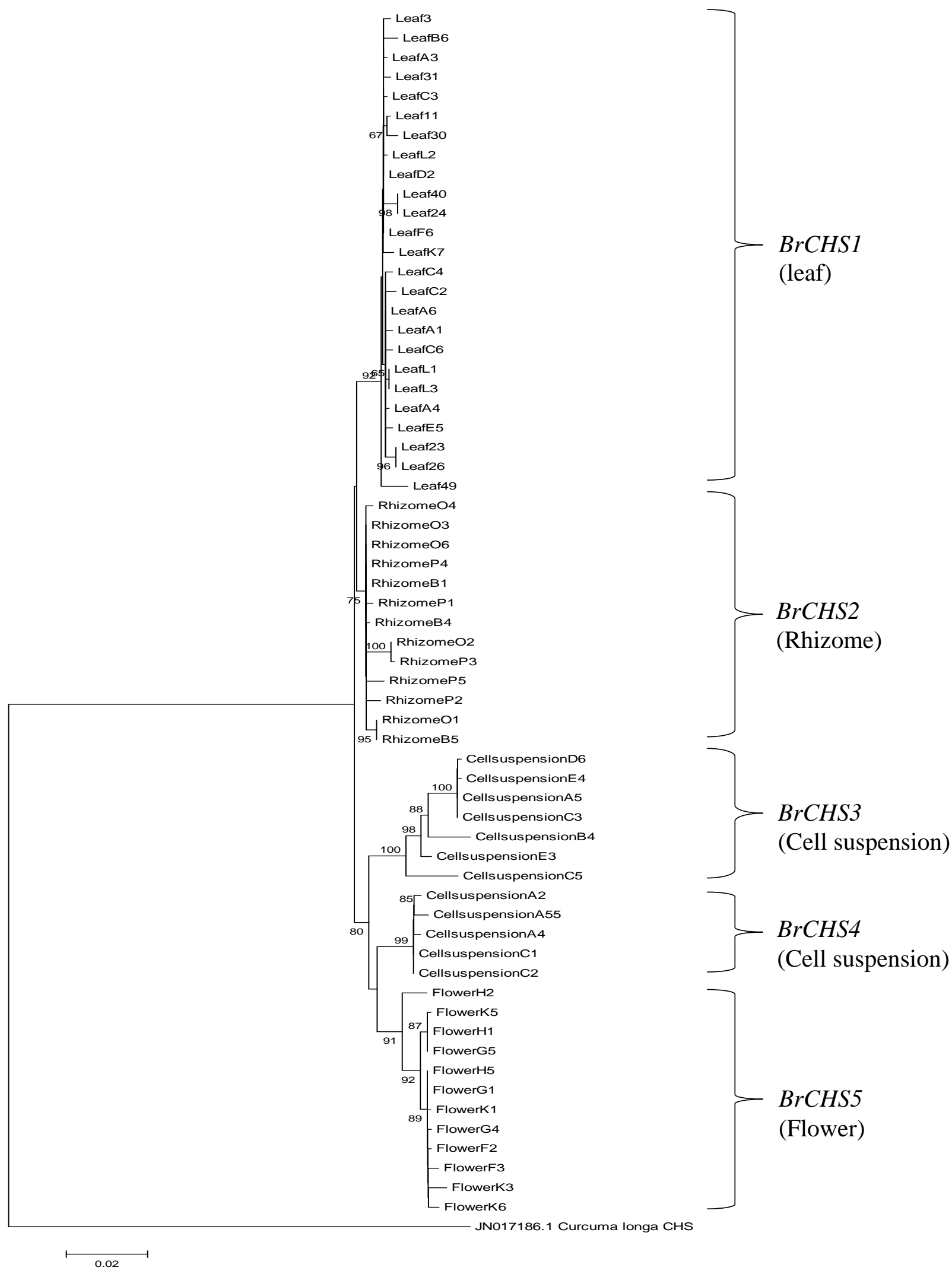

Figure 2. The phylogenetic tree was inferred using the Neighbor-Joining method based on deduced amino acid sequence. CHS from Curcuma longa (JN017186.1) was used as an outgroup. The percentage of replicate trees in which the associated taxa clustered together in the bootstrap test (1000 replicates) was shown above the branches. 
In addition, the representative of $\mathrm{BrCHS}$ transcript also aligned with $\mathrm{CHS}$ gene of some other plants extracted from GenBank database. Amino acids from five $\mathrm{BrCHS}$ transcripts from $\mathrm{B}$. rotunda were clustered together with other monocots in Zingiberales Order and most closely related to Curcuma genus ( $C$. longa and $C$. alismatifolia) as shown in Suppl. 3. Comparative analysis also revealed that the deduced amino acid sequence of $\mathrm{BrCHS}$ was highly homologous to $C H S$ gene from other monocotyledonous plant species. For example, $M$. acuminata (87-89\%), $O$ sativa (81-82\%), C. longa (86-89\%), C. alismatifolia (84-88\%), Z. mays (79-82\%) and $M$. sativa (79-82\%). Since there were four to five transcripts identified, the presence of four to five copies of BrCHSs was suggested; southern blot analysis was performed to corroborate the result. This analysis revealed that four to five bands can be clearly observed after genomic DNA digestion with DraI and EcoRV, respectively, as shown in Figure 3.

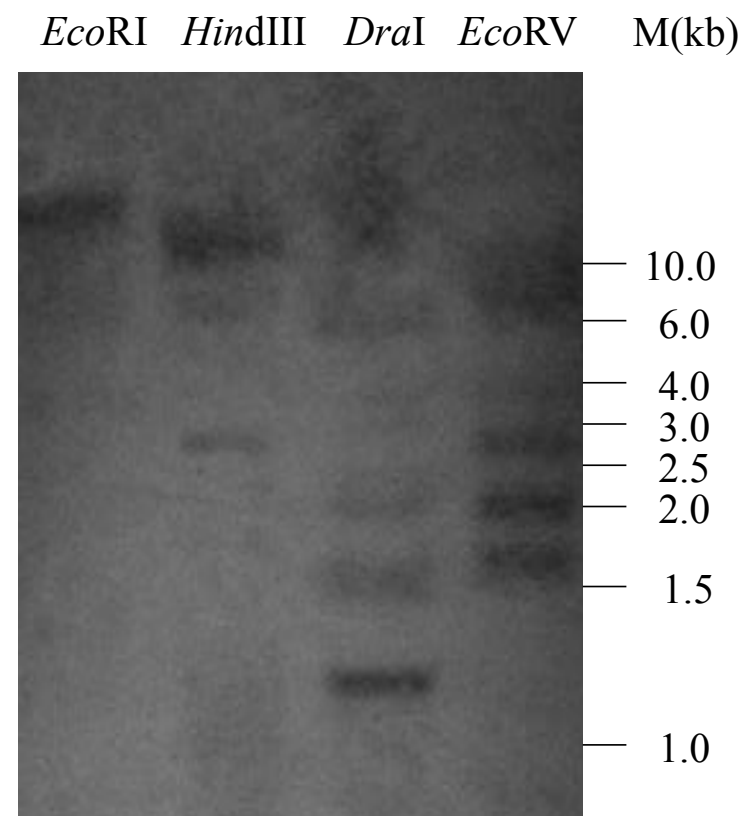

Figure 3. The copy number determination was done by digesting genomic DNA with four different restriction enzymes, EcoRI, HindIII, DraI and EcoRV. A minimal of four and five prominent bands was observed for DraI and EcoRV digested genomes, respectively.

Cell suspension cultures were
transformed using Agrobacterium-
mediated method, and cells were then
grown on semi-solid media and further
verified by their callus morphology, and
pre-screened for $\beta$-glucuronidase (GUS)

histochemical analysis. There was no observable phenotypic difference between wildtype and transgenic calli observed after several months of subculture (data not shown). Meanwhile, after a few rounds of hygromycin selection, 125 resistant calli 
were selected and pre-screened for $\beta$ glucuronidase (GUS) histochemical analysis. About $65 \%$ resistant calli (81/125) showed positive signals in GUS staining with different degrees of GUS staining intensities, and 10 clumps of them were selected and transferred to MS liquid media and cultured for two months to develop a stable line of ten transgenic cell suspension cultures. A GUS assay was applied after the harvest, showing that recombinant $\mathrm{BrCHS} 2$ transcript was stably expressed in all ten transgenic lines compared to wildtype (Figure 4).

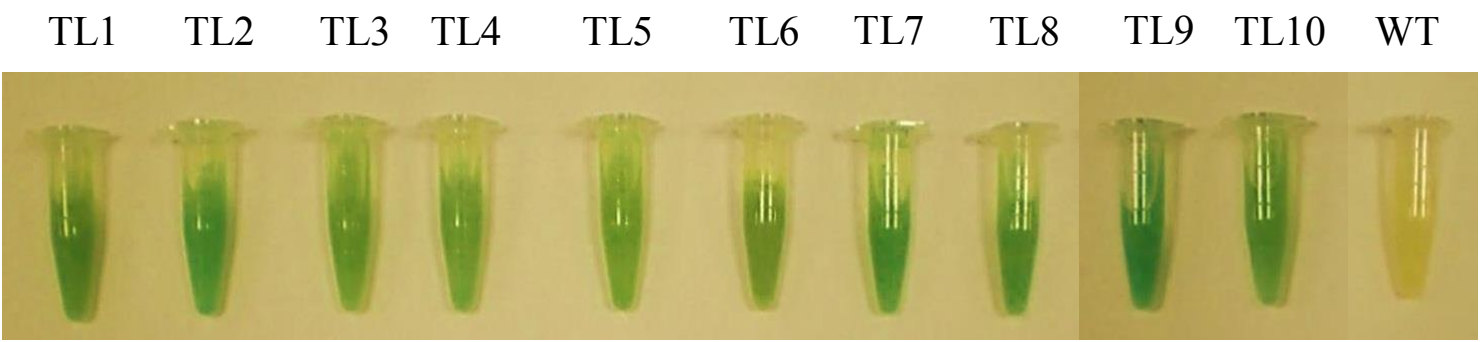

Figure 4. After harvested, two-month-old suspension cultures were assayed with GUS and showed positive greenish blue intensity in all ten transgenic lines (TL1-10) compared to wildtype (WT).

Successful transformation of recombinant $\mathrm{BrCHS} 2$ transcript was further confirmed by multiplex PCR analysis. In each line, the recombinant $\mathrm{BrCHS} 2$, HptII and EFla genes were successfully amplified with $1.3 \mathrm{~kb}, 0.7 \mathrm{~kb}$ and $0.2 \mathrm{~kb}$, respectively (Figure 5). The selection of transgenic suspension cultures was based on the presence of HptII which detoxified the aminocyclitol antibiotic hygromycin B. Meanwhile, EFI $\alpha$ was a housekeeping gene involved in basic functions needed for the sustenance of the cell, and constitutively expressed in plants.

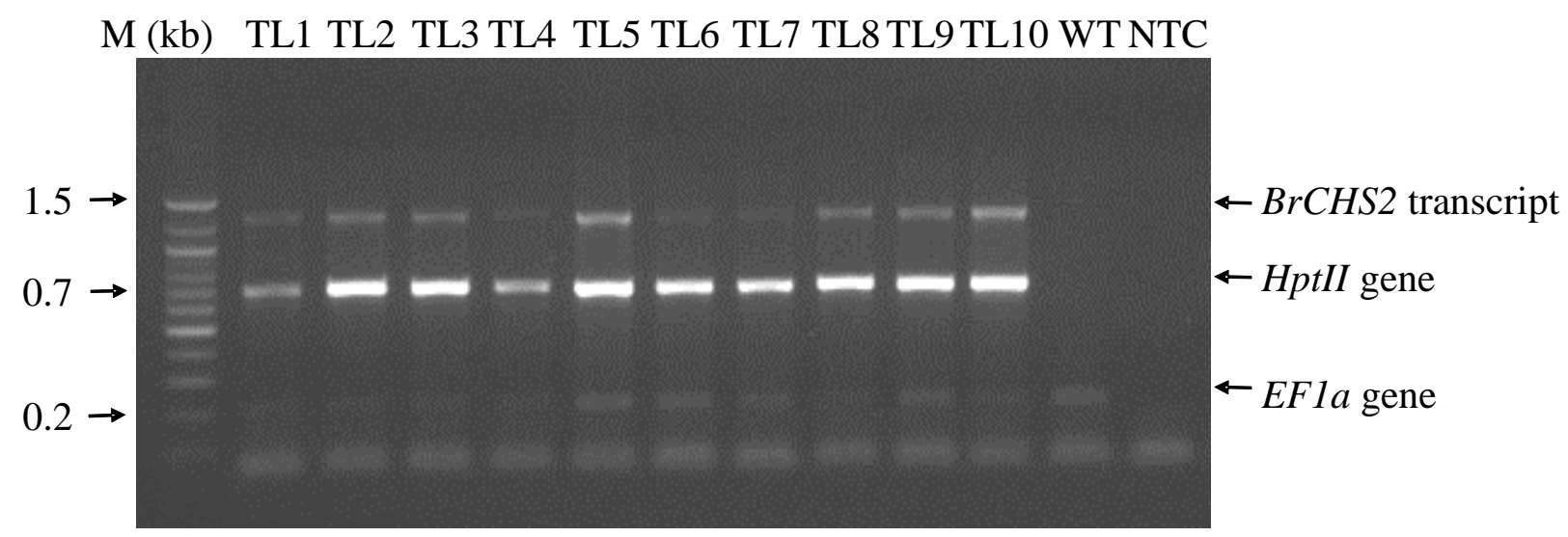

Figure 5. Multiplex PCR analysis showed all ten transgenic suspension cultures harbouring all three genes. No amplified products were observed except for EF $1 \alpha$ in wildtype (WT).

Lane M: 100bp ladder, Lanes TL1-10: Ten transgenic suspension cultures, NTC: NonTemplate 
Next, to observe the BrCHS expression levels, RT-qPCR was evaluated, and about 2 to 27-fold higher expression level than wildtype line was determined (Figure 6). Eight out of ten transgenic suspension cultures; TL1 (9.62-fold), TL2 (9.63-fold), TL3 (8.14-fold), TL5 (8.10fold), TL6 (5.61-fold), TL7 (5.35-fold),
TL9 (27.49-fold) and TL10 (19.74-fold) showed higher $\mathrm{BrCHS}$ expression level than wildtype at different significance levels (not significant in two lines, $p<0.01$ in two lines and $p<0.001$ in six lines). Meanwhile, TL4 and TL8 expressed 2.76fold and 1.93-fold slightly higher than wildtype.

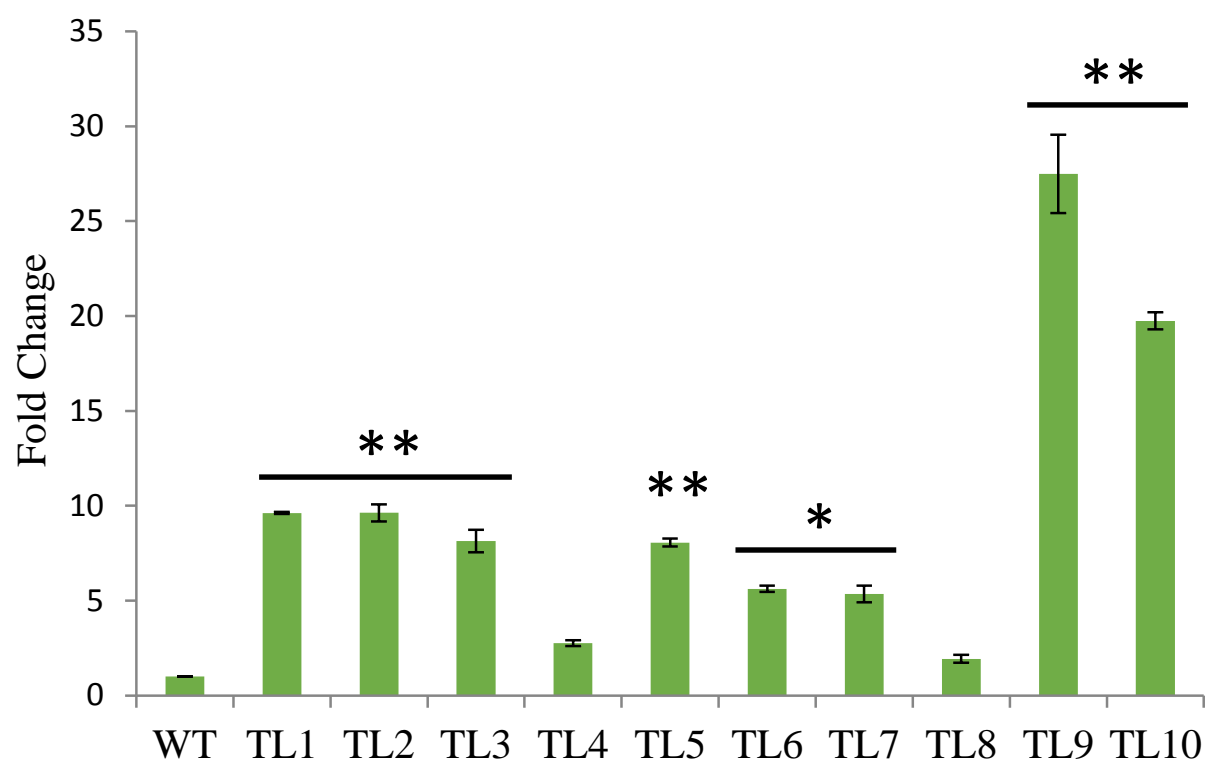

Figure 6. Ten transgenic lines (TL1-10) showed different expression levels (2 to 27-fold change) of $\mathrm{BrCHS}$ compared to wildtype (WT). The expression was normalized with elongation factor gene, EF $1 \alpha$. * Significant at $\mathrm{P}<0.01$, ** Significant at $\mathrm{P}<0.001$

\section{DISCUSSIONS}

In this study, the evaluation on the key enzyme, chalcone synthase (CHS) was established as this enzyme was responsible for catalysing a wide variety production of secondary metabolites. In an effort to investigate the expression of chalcone synthase $(\mathrm{CHS})$ transcripts and its overexpression in cell suspension cultures of Boesenbergia rotunda, the study was first designed to characterize more than 62 $\mathrm{BrCHS}$ clones from four different parts of $B$. rotunda plants. BrCHS protein comprised of an active amino acid residues that were highly conserved and resided at the second exon. Between the active site cavities, there were two conserved "gatekeeper" phenylalanine's (Phe-215 and Phe-265) connected with CoA-binding tunnel. The existence of these two "gatekeepers" facilitated decarboxylation of malonyl-CoA, and was involved in the condensation reactions of CHS (Abe et al., 2003; Jez et al., 2002).

Five $\mathrm{BrCHS}$ transcripts were predominantly expressed and differently 
clustered in the phylogenetic analysis. This characterization constituted multiple copies, supported by a variety of copy number that has also been reported in several plant species. For example, plant species such as Gerbera hybrida (Deng et al., 2014), Morus atropurpurea (Wang et al., 2017), Sorghum bicolor (Lo et al., 2002) and Ipomea purpurea (Durbin et al., 2000) which contain small number of $C H S$ gene families of 3, 5, 6 and 7, respectively. Our results also corroborated with southern blot analysis where at least four to five clear bands were observed after treated with DraI and $E c o \mathrm{RV}$ indicating that there were more than one copy of $B r C H S$ existed in the $B$. rotunda plant genome.

Since there were multiple copies of $B r C H S$ detected in B. rotunda plants, this study also revealed high degree of similarity among five representatives of BrCHS transcripts. This finding was supported by earlier studies, for example, $92.3 \%$ to $100 \%$ of $\mathrm{CHS}$ sequence similarity as reported in Triticum aestivum (Trojan et al., 2014) and in Gerbera hybrida, which shared $80.0 \%$ to $88.0 \%$ of CHS amino acid sequence identity (Deng et al., 2014). There were nine copies of $\mathrm{CHS}$ genes detected in Glycine max; however, only CHS7 and CHS8 had contributed to most CHS transcripts, and shared $97.0 \%$ similarity at the nucleotide level (Tuteja et al., 2004). The occurrence of high similarity among BrCHS transcripts indicated a strong conservation of the structure and active site residues that are important during the synthesis of plant metabolites, and thus being a typical CHS protein. BrCHS transcripts have formed separate clusters, and showed tissue-specific expression pattern. Even though there was no confirmation on $\mathrm{BrCHS}$ expression level in every part of $B$. rotunda plants using RTqPCR approach, our results were in accordance with a previous study. For example, spatial regulation of the $C H S$ genes in different tissues was previously detected especially in the roots of Polygonum minus ranging approximately from 10 to 15 -fold higher compared to the leaves and stems (Roslan et al., 2013).

A possible explanation for tissuespecific expression may be due to the differences in the structural sequence diversity of the cis-acting element within all five $\mathrm{BrCHS}$ promoters which controlled the gene regulation at the promoter level. Previous study reported that the CHS gene promoter has a complex series of regulator cis-acting elements localized upstream of the transcriptional start (Hernandez-Garcia \& Finer, 2014). According to a report by Faktor et al. (1997), a 39 bp motif is identified which describes the two adjacent motifs, the G-Box (CACGTG) and the HBox (CCTACC), that are often found near a TATA Box in the promoter of CHS15 in Phaseolus vulgaris. The role of these motifs is for specific expression in flowers and roots of Phaseolus vulgaris, and their role is described as a tissue-specific regulatory element. Thus, it might be possible that the gene expression patterns among five $\mathrm{BrCHS}$ transcripts are due to the sequence variation and regulatory position that have been mapped between the two adjacent motifs, or possessed a complex structure of the motif(s) in the promoter region. Hence, taking $\mathrm{BrCHS} 2$ transcript as an example, the complex interplay between different cis-elements in $\mathrm{BrCHS} 2$ promoter may be crucial for its expression specifically in $B$. rotunda's rhizome compared to other parts of the plant.

Therefore, in order to develop a full picture of $\mathrm{BrCHS}$ expression pattern and to modulate this important transcript, additional studies on tissue specific 
expression as well as functional dissection of $B$. rotunda's $C H S$ promoter were needed to delineate the function of each transcript. These strategies further revealed the sequence motifs which are essential for regulating the $\mathrm{BrCHS}$ transcript in specific tissue. Our study on differentiating BrCHS expression profiles in specific tissue of $B$. rotunda plants cannot be achieved since there was a high sequence similarity among the $\mathrm{BrCHS}$ transcript. It was imperative to obtain quantitative data using a more sensitive and powerful technique by designing gene specific primers and probes for TaqMan RT-qPCR in order to perform an in-depth investigation on each of the BrCHS transcript.

The transformation of monocot plants using Agrobacterium-mediated method gained popularity when a large number of transgenic monocots were successfully produced, and the function of the inserted gene was extensively studied (Éva et al., 2008; Reyes et al., 2010; Zhang et al., 2013; Hiei et al., 2014). Therefore, with the successful and efficient method of using Agrobacterium as a way for gene transfer in several monocot plants, transferring BrCHS2 in B. rotunda plant genome would not be a problem due to better understanding of the monocot transformation process and availability of the vectors for efficient gene transfer (Komori et al., 2008). Agrobacteriummediated transformation has become a viable and useful tool for the genetic engineering in monocots of $B$. rotunda which would later drive the synthesis of important metabolite compounds in the flavonoid biosynthesis pathway. Additionally, with this information, engineering a desirable metabolic pathway in $B$. rotunda will be more straightforward and on target.

\section{CONCLUSIONS}

Overall, there are at least four to five $\mathrm{BrCHS}$ transcripts which are successfully characterized and detected in different parts of $B$. rotunda plants. The idea on overexpressing $\mathrm{BrCHS} 2$ (isolated from rhizomes) could give a clue to exploit the synthesis of targeted compounds and this research has laid the foundation for future exploration in $B$. rotunda. Future research could also be conducted to determine the effect on silencing the $\mathrm{BrCHS}$ itself, adding more copies of the other downstream or potential genes involved in the pathway that may lead to metabolite production. More information on changes in gene expression level would help to establish a greater degree of accuracy on this matter.

\section{ACKNOWLEDGEMENTS}

We hereby acknowledge the financial supports from University of Malaya through these research grants FL 002-020 and PV085-2012.

\section{REFERENCES}

Abe, I., Sano, Y., Takahashi, Y., \& Noguchi, H. (2003). Site-directed mutagenesis of benzalacetone synthase: The role of PHE 215 in plant type III polyketide synthases. Journal of Biological Chemistry, 278(27), 25218-25226.

Anguraj Vadivel, A. K., Krysiak, K., Tian, G., \& Dhaubhadel, S. (2018). Genome-wide identification and localization of chalcone synthase family in soybean (Glycine $\max$ [L] Merr). BMC Plant Biology, 18(1), 325. 
Austin, M. B., \& Noel, J. P. (2003). The chalcone synthase superfamily of type III polyketide synthases. Natural Product Reports, 20(1), 79-110.

Ayabe, S., Uchiyama, H., Aoki, T., \& Akashi, T. (2010). Plant Phenolics: Phenylpropanoids. Comprehensive Natural Products II, 929-976.

Bhamarapravati, S., Juthapruth, S., Mahachai, W., \& Mahady, G. (2006). Antibacterial activity of Boesenbergia rotunda (L.) mansf. and Myristica Fragrans houtt. against helicobacter pylori. Songklanakarin Journal of Science and Technology, 28(suppl. 1), 157163.

Chan, K. L., Ho, C. L., Namasivayam, P., \& Napis, S. (2007). A simple and rapid method for RNA isolation from plant tissues with high phenolic compounds and polysaccharides. Protocol Exchange, 1. Retrieved from http://www.nature.com/protocolexc hange/protocols/208

Cheenpracha, S., Karalai, C., Ponglimanont, C., Subhadhirasakul, S., \& Tewtrakul, S. (2006). Anti-HIV-1 protease activity of compounds from Boesenbergia pandurata. Bioorganic and Medicinal Chemistry, 14(6), 1710-1714.

Deng, X., Bashandy, H., Ainasoja, M., Kontturi, J., Pietiäinen, M., Laitinen, R. A., ... Teeri, T. H. (2014). Functional diversification of duplicated chalcone synthase genes in anthocyanin biosynthesis of Gerbera hybrida. New Phytologist, 201, 1469-1483.
Deng, Y., Li, C., Li, H., \& Lu, S. (2018). Identification and characterization of flavonoid biosynthetic enzyme genes in Salvia miltiorrhiza (Lamiaceae). Molecules, 23(6), 1467.

Durbin, M. L., McCaig, B., \& Clegg, M. T. (2000). Molecular evolution of the chalcone synthase multigene family in the morning glory genome. Plant Molecular Biology, 42(1), 79-92.

Éva, C., Csóti, I., \& Tamás, L. (2008). Agrobacterium-mediated barley transformation. Acta Biologica Szegediensis, 52(1), 49-51.

Falcone Ferreyra, M. L., Rius, S. P., \& Casati, P. (2012). Flavonoids: biosynthesis, biological functions, and biotechnological applications. Frontiers in plant science, 3, 222.

Faktor, O., Loake, G., Dixon, R. A., \& Lamb, C. J. (1997). The G-box and H-box in a $39 \mathrm{bp}$ region of a French bean chalcone synthase promoter constitute a tissue-specific regulatory element. Plant Journal, 11(5), 1105-1113.

Feinbaum, R. L., \& Ausubel, F. M. (1992). Transcriptional Regulation of the Arabidopsis thaliana Chalcone Synthase Gene. Molecular and Cellular Biology, 8(5), 1985-1992.

Ferrer, J. L., Jez, J. M., Bowman, M. E., Dixon, R. A., \& Noel, J. P. (1999). Structure of chalcone synthase and the molecular basis of plant polyketide biosynthesis. Nature Structural Biology, 6(8), 775-784.

Han, Y., Ding, T., Su, B., \& Jiang, H. (2016). 
identification, characterization and expression analysis of the chalcone synthase family in maize. International Journal of Molecular Sciences, 17(2), 161.

Hernandez-Garcia, C. M., \& Finer, J. J. (2014). Identification and validation of promoters and cis-acting regulatory elements. Plant Science, 217-218, 109-119.

Hiei, Y., Ishida, Y., \& Komari, T. (2014). Progress of cereal transformation technology mediated by Agrobacterium tumefaciens. Frontiers in Plant Science, 5, 1-11.

Jez, J. M., Bowman, M. E., \& Noel, J. P. (2002). Expanding the biosynthetic repertoire of plant type III polyketide synthases by altering starter molecule specificity. Proceedings of the National Academy of Sciences of the United States of America, 99(8), 53195324.

Khayat, E., Duvdevani, A., Lahav, E., \& Balesteros, B. A. (2004). Somaclonal variation in banana (Musa acuminate cv. Grande Naine): genetic mechanism, frequency and application as a tool for clonal selection. In S. M. Jain \& R. Swennen (Eds.), Banana improvement: cellular, molecular biology and induced mutation. Science, 97-109.

Kiat, S.K., Pippen, R., Yusof, R., Ibrahim H., Khalid, N., Rahman, N.A. (2006) Inhibitory activity of cyclohexenyl chalcone derivaties and flavonoids of fingerroot, Boesenbergia rotunda (L.), towards dengue-2 virus NS3 protease.
Bioorganic \& Medicinal Chemistry Letters, 16, 3337-3340.

Kirana, C., Jones, G. P., Record, I. R., \& McIntosh, G. H. (2007). Anticancer properties of panduratin A isolated from Boesenbergia pandurata (Zingiberaceae). Journal of Natural Medicines, 61, 131-137.

Komori, T., Takakura, Y., Ueld, J., Kato, N., Ishida, Y., \& Hiei, Y. (2008). Binary vectors and super binary vectors. In Methods in Molecular Biology (Vol. 343, pp. 15-42).

Kreuzaler, F., Ragg, H., Fautz, E., Kuhn, D. N., \& Hahlbrock, K. (1983). UVinduction of chalcone synthase mRNA in cell suspension cultures of Petroselinum hortense. Proceedings of the National Academy of Sciences of the United States of America, 80(9), 2591-3.

Lo, C., Coolbaugh, R. C., \& Nicholson, R. L. (2002). Molecular characterization and in silico expression analysis of a chalcone synthase gene family in Sorghum bicolor. Physiological and Molecular Plant Pathology, 61(3), 179-188.

Murashige, T. \& Skoog, F. (1962). A revised medium for rapid growth and bioassays with tobacco tissue cultures. Physiologia Plantarum, 15, 473-497.

O'Leary, N. A., Wright, M. W., Brister, J. R., Ciufo, S., Haddad, D., McVeigh, R., .. Pruitt, K. D. (2016). Reference sequence (RefSeq) database at NCBI: current status, taxonomic expansion, and functional annotation. Nucleic 
Acids Research, 44(D1), D733D745.

Rasche, S., Herwartz, D., Schuster, F., Jablonka, N., Weber, A., Fischer, R., \& Schillberg, S. (2016). More for less: Improving the biomass yield of a pear cell suspension culture by design of experiments. Scientific Reports, 6, 23371.

Resmi, M.S. \& Soniya, E.V. (2012) Molecular cloning and differential expressions of two cDNA encoding Type III polykektide synthase in different tissues of Curcuma longa L. Gene, 491, 278-283.

Reyes, F. C., Sun, B., Guo, H., Gruis, D. F., \& Otegui, M. S. (2010). Agrobacterium tumefaciensmediated transformation of maize endosperm as a tool to study endosperm cell biology. Plant Physiology, 153, 624-631.

Roslan, N. D., Tan, C., Ismail, I., \& Zainal, Z. (2013). cDNA cloning and expression analysis of the chalcone synthase gene ( $\mathrm{CHS}$ ) from Polygonum minus. Australian Journal of Crop Science, 7(6), 777783.

Sambrook, J., Fritsch, E. F., and Maniatis, T. (1989). Molecular Cloning: A Laboratory Manual. Cold Spring Harbor, NY: Cold Spring. Harbor Laboratory Press.

Shashank, K., \& Abhay, K. (2013). Review Article Chemistry and Biological Activities of Flavonoids: An Overview. The Scientific World Journal, 4(2), 32-48.

Shindo, K., Kato, M., Kinoshita, A., Kobayashi, A., \& Koike, Y. (2006). Analysis of antioxidant activities contained in the Boesenbergia pandurata Schult. rhizome. Bioscience, Biotechnology, and Biochemistry, 70(9), 2281-2284.

Suh, D. Y., Fukuma, K., Kagami, J., Yamazaki, Y., Shibuya, M., Ebizuka, Y., \& Sankawa, U. (2000). Identification of amino acid residues important in the cyclization reactions of chalcone and stilbene synthases. The Biochemical Journal, 350 Pt 1(Pt 1), 229-235.

Sun, W., Meng, X., Liang, L., Jiang, W., Huang, Y., He, J., ... Wang, L. (2015). Molecular and biochemical analysis of chalcone synthase from freesia hybrid in flavonoid biosynthetic pathway. PLOS ONE, 10(3), 1-18.

Tai, D., Tian, J., Zhang, J., Song, T., \& Yao, Y. (2014). A Malus crabapple chalcone synthase gene, $M c C H S$, regulates red petal color and flavonoid biosynthesis. PLOS ONE, 9(10), 1-13.

Tewtrakul, S., Subhadhirasakul, S., Karalai, C., Ponglimanont, C., \& Cheenpracha, S. (2009). Antiinflammatory effects of compounds from Kaempferia parviflora and Boesenbergia pandurata. Food Chemistry, 115(2), 534-538.

Trojan, V., Musilová, M., Vyhnánek, T., Klejdus, B., Hanáček, P., \& Havel, L. (2014). Chalcone synthase expression and pigments deposition in wheat with purple and blue colored caryopsis. Journal of Cereal Science, 59(1), 48-55.

Tuteja, J. H., Clough, S. J., Chan, W. C., \& Vodkin, L. O. (2004). Tissuespecific gene silencing mediated by a naturally occurring chalcone synthase gene cluster in Glycine 
max. The Plant Cell, 16(4), 819835.

Wang, C., Zhi, S., Liu, C., Xu, F., Zhao, A., Wang, X., Tang, X., Li, Z., Huang, P., Yu, M. (2017). Isolation and characterization of a novel chalcone synthase gene family from mulberry. Plant Physiology and Biochemistry, 115, 07-18.

Wang, Z., Yu, Q., Shen, W., El Mohtar, C. A., Zhao, X., \& Gmitter, F. G. Jr. (2018). Functional study of CHS gene family members in citrus revealed a novel CHS gene affecting the production of flavonoids. BMC Plant Biology, 18(1), 189.

Wani, T. A., Pandith, S. A., Gupta, A. P., Chandra, S., Sharma, N., \& Lattoo, S. K. (2017). Molecular and functional characterization of two isoforms of chalcone synthase and their expression analysis in relation to flavonoid constituents in Grewia asiatica L. PloS ONE, 12(6), e0179155.

Wannapinpong, S., Srikulnath, K., Thongpan, A., Choowongkomon,
K., \& Peyachoknagul, S. (2013). Molecular cloning and characterization of theCHS gene family in turmeric (Curcuma longa Linn.). Journal of Plant Biochemistry and Biotechnology, 24(1), 25-33.

Wong, S. M., Salim, N., Harikrishna, J. A., \& Khalid, N. (2013). Highly efficient plant regeneration via somatic embryogenesis from cell suspension cultures of Boesenbergia rotunda. In Vitro Cellular and Developmental Biology - Plant, 49(6), 665-673.

Yusuf, N. A., Suffian, M., Annuar, M., \& Khalid, N. (2013). Existence of bioactive flavonoids in rhizomes and plant cell cultures of Boesenbergia rotunda (L.) Mansf. Kulturpfl. Australian Journal of Crop Science, 7(6), 730-734.

Zhang, W. J., Dewey, R. E., Boss, W., Phillippy, B. Q., \& Qu, R. (2013). Enhanced Agrobacterium-mediated transformation efficiencies in monocot cells is associated with attenuated defense responses. Plant Molecular Biology, 81(3), 273-286.

Supplementary 1. Primers' sequences that were used in this study

\begin{tabular}{|c|c|}
\hline Primers & Sequence $\left(5^{\prime}-3^{\prime}\right)$ \\
\hline GeneRacer $^{\mathrm{TM}} 5$ & 5' - CGACTGGAGCACGAGGACACTGA - 3' \\
\hline GeneRacer $^{\mathrm{TM}}$ Oligo dT & 5' - GCTGTCAACGATACGCTACGTAACGG \\
\hline & CATGACAGTG(T) $)_{24}-3$ \\
\hline CHS_intR & 5' - CTTGTCGCACATTCTTTTAAACTTCTCC - 3' \\
\hline CHS_F & 5'-AACAGCCATGGCCAAAGTCCAGGAGATC-3' \\
\hline CHS_R & 5'-GTTCGACTAGTATGATTGATTGGCTTGC-3’' \\
\hline pCAMBIA_R & 5'-TTGTGCCCATTAACATCAC-3' \\
\hline hptII_F & 5'-CAAGACCTGCCTGAAACCGA-3' \\
\hline hptII_R & 5'-TCGGCGAGTACTTCTACACA-3' \\
\hline CHS_rt_F & 5'-ACGCTGACTACTACTTCC-3' \\
\hline CHS_rt_R & 5'-GGGTTCTCCTTCAGAATC-3' \\
\hline EF1a_F & 5'-GAGGCTGGTATCTCAAAGG-3' \\
\hline $\mathrm{EF} 1 \alpha \mathrm{R}$ & 5'-GTGCCTTTGAGTACTTTGG-3' \\
\hline
\end{tabular}


Supplementary 2. Amino acid alignment of BrCHSs and other plant's CHS

Alignment of CHS deduced amino acid sequences from B. rotunda (leaf, rhizome, flower and suspension cultures), Cucurma longa (ClPKS9; JN017186.1), Curcuma alismatifolia (CaCHS; GU140082.1), Medicago sativa (MsCHS2; L02902.1 Musa acuminata (MaPKSIII3; GU724609.1), Oryza sativa (OsCHS;
AB000801.2) and Zea mays (ZmCHS; NM_001155550.1). The amino acids of the catalytic residue Cys164-His303-Asn336 triads were marked by green boxes. Meanwhile, the blue box represents Pro375 and GFGP375G loop and the red box represents active site residues for CHS. Bold sequence represents the Phe215 and Phe265.
\#BrCHS1 \#BrCHS2 \#BrCHS 3 \#BrCHS 4 \#BrCHS5 \#ClPKS \#CaCHS \#MSCHS2 \#MaPKSIII3

\#OSCHS

\#ZmCHS

\#BrCHS1 \#BrCHS2

\#BrCHS3

\#BrCHS

\#BrCHS5

\# $\mathrm{B}$ C $\mathrm{CH}$ PKS 9

\#CIPKS

\#CaCHS

\#MaPKSIII3

\#OSCHS

\#ZmCHS

\#BrCHS1

\#BrCHS2

\#BrCHS3

\#BrCHS 4

\#BrCHS 5

\#CIPKS 9

\#CaCHS

\#MSCHS2

\#MaPKSIII 3

\#OSCHS

\#OSCHS

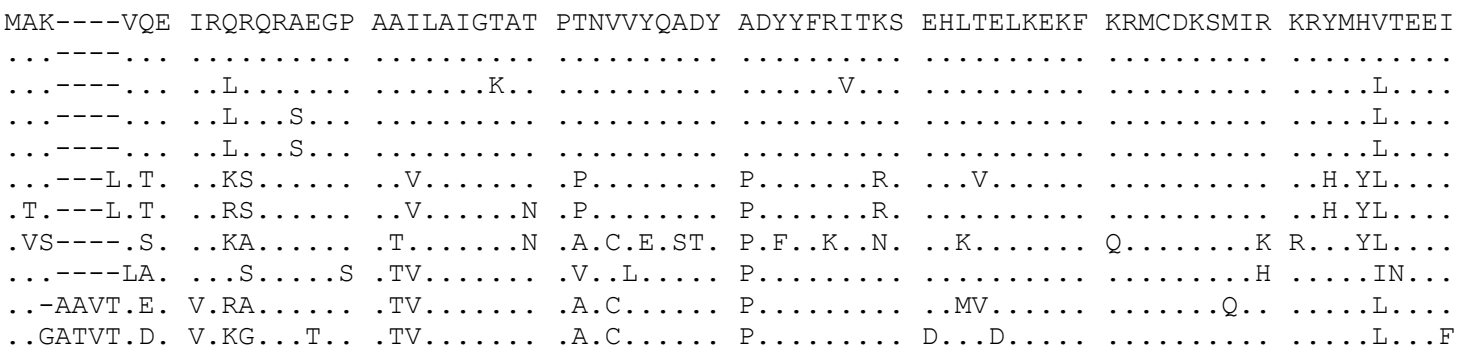

LKENPNMCAY MEPSLDERQD IVVVEVPKLG KEAAAKAIKE WGQPKSKITH LIVCTTSGVD MPGADYQITK LLGLRPSVNR

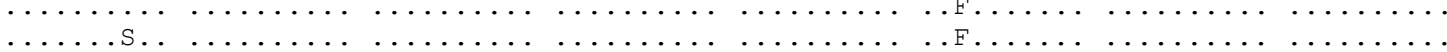

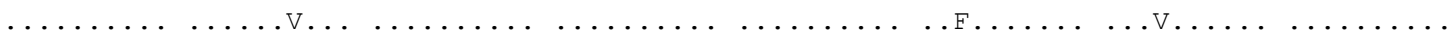

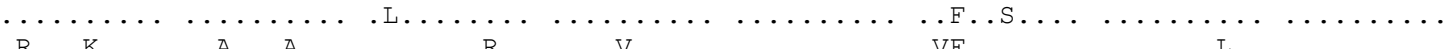

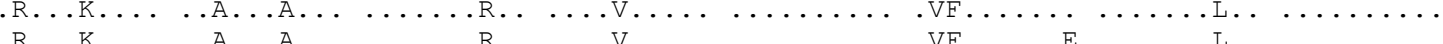

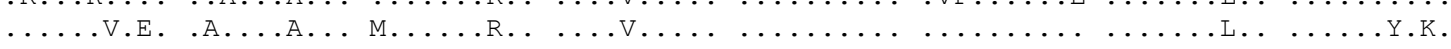

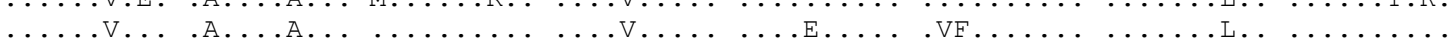

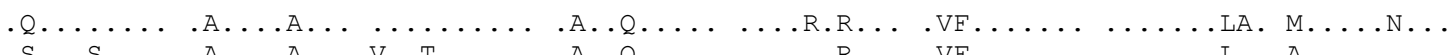

. S...S $\ldots . . . . . . .$.
FMMYQQGCEA GGTVLRLAKD LAENNRGARV LVVCSEITAV TFRGPSESHL DSLVGQALFG DGAGAIIVGA DPDLEIERPL

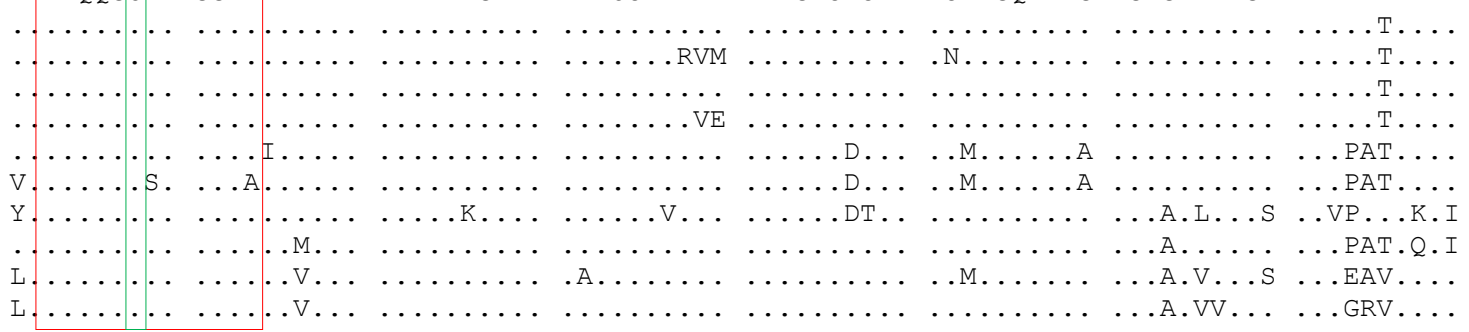


\#BrCHS1

\#BrCHS2

\#BrCHS

\#BrCHS 4

\#BrCHS5

\#CIPKS

\#CaCHS

\#MsCHS2

\#MaPKSIII 3

\#OSCHS

\#ZmCHS

\#BrCHS1

\#BrCHS2

\#BrCHS3

\#BrCHS 4

\#BrCHS5

\#ClPKS 9

\#CaCHS

\#MsCHS2

\#MaPKSIII 3

\#OSCHS

\#ZmCHS
FELVSASQTI LPDSEGAIDG HLREVGLTFH LLKDVPGLIS KNIEKSLVEA FAPLGIDDWN SLFWIAHPGG PAILDQVEAK

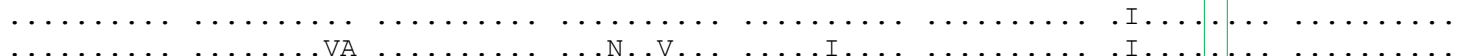

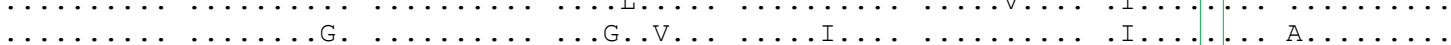

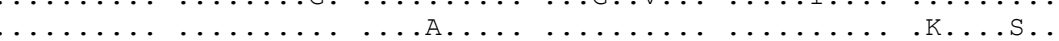

.

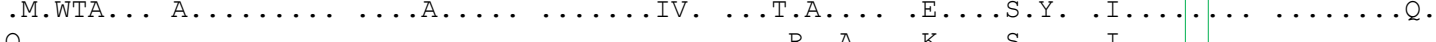

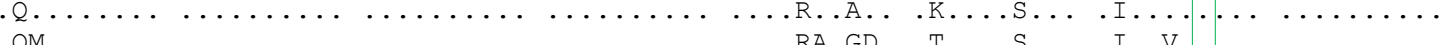

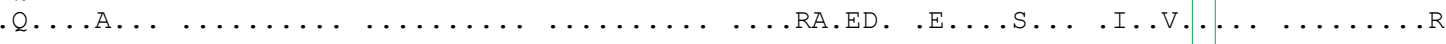

LALEKEKMAA TRQVLSEYGN MSSACVIFIL DEMRRKSAQE GKTTTGEGLN WGVLFGFGP LTVETVVLHS KPINH*---

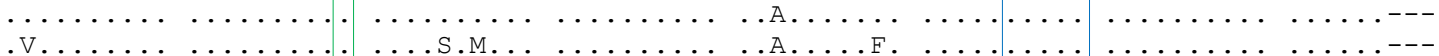

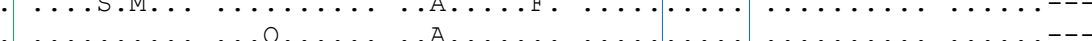

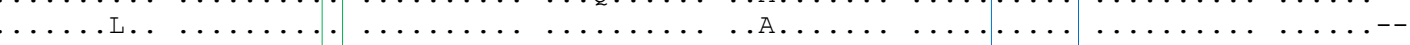

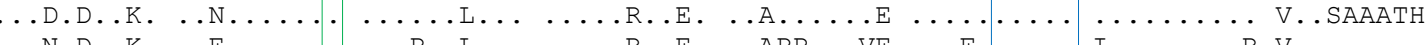

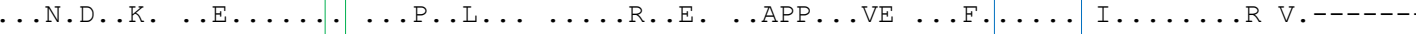

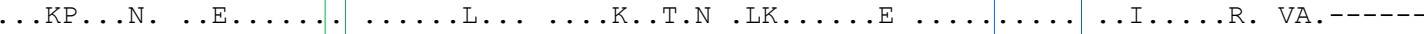

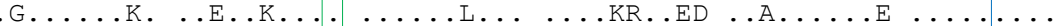
GG D.R R . H

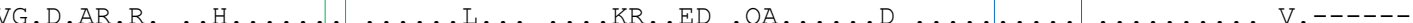


Supplementary 3. Phylogenetic tree among BrCHS and other plant's CHS

The phylogenetic tree was inferred using the Neighbor-Joining method. Alignment of deduced amino acid sequences was performed among five $\mathrm{BrCHSs}$ from $B$. rotunda plants (BrCHS1, BrCHS2, $\mathrm{BrCHS3}, \mathrm{BrCHS4}$ and $\mathrm{BrCHS5)}$ and CHS in various monocot plants; Cucurma longa (ClPKS9; JN017186.1), Curcuma alismatifolia (CaCHS; GU140082.1), Musa acuminata (MaPKSIII3; GU724609.1), Oryza sativa (OsCHS; AB000801.2), Zea mays (ZmCHS; NM_001155550.1) and Medicago sativa (MsCHS2; L02902.1). The numbers above the branches refer to the bootstrap values.

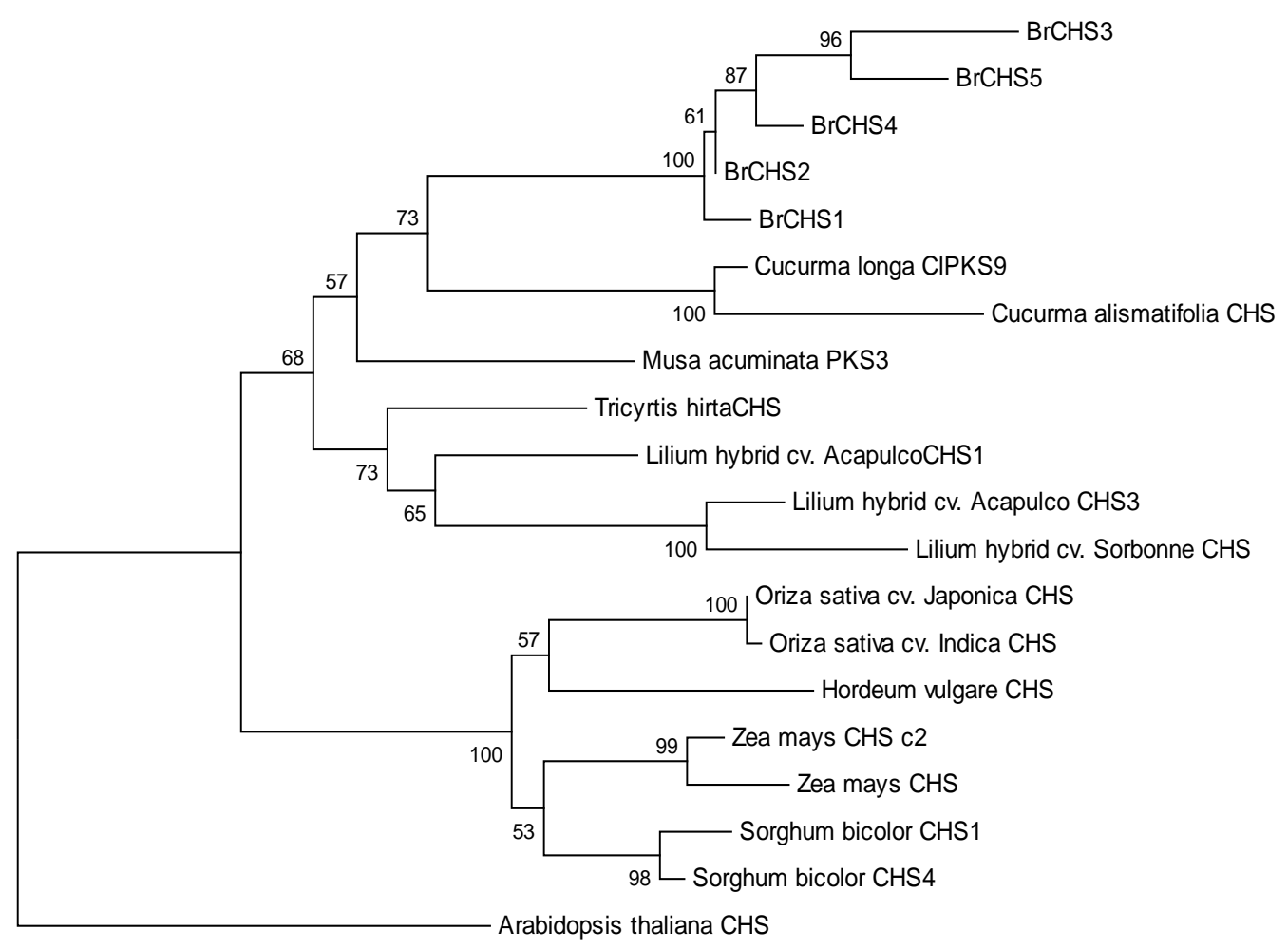

0.02 